\title{
Equalization Techniques for Time Varying Channels in MIMO based Wireless Communication
}

\author{
T Padmavathi ${ }^{1}$ and Varghese Thattil ${ }^{2}$ \\ ${ }^{1}$ CVR College of Engineering, Department of ECE, Ibrahimpatan, R.R.District, A.P., India \\ Email:padmatp41@gmail.com \\ ${ }^{2}$ CVR College of Engineering, Department of ECE, Ibrahimpatan, R.R.District, A.P., India \\ Email:mailthattil@gmail.com
}

\begin{abstract}
In mobile integrated service digital network, high bit-rate data transmission is essential for many services such as video, multimedia etc. When data is transmitted at high bit-rate, wireless channels exhibit delay dispersion because of multipath components (MPC). Different propagation times from transmitter (Tx) to receiver $(\mathbf{R x})$ in MIMO systems produces different MPCs. This in turn leads to Inter Symbol Interference (ISI), which can greatly disturb the transmission of digital signals. If delay spread is smaller than the symbol duration, it can lead to considerable Bit Error Rate (BER) degradation. Equalization defines any signal processing technique used at receiver to make the signal less susceptible to delay spread. Equalizers require an estimate of channel impulse or frequency response to mitigate the ISI. In this paper the performance of equalization techniques are compared by considering 2 transmit and 2 receive antennas (resulting $2 \times 2$ MIMO Channel). Assume that channel is flat fading Rayleigh multipath- channel and modulation is BPSK.
\end{abstract}

Index terms- Delay spread, Inter Symbol Interference, Rayleigh multipath channel, Adaptive equalization

\section{INTRODUCTION}

Wireless communication has been optimized due to growth of cellular Telephone and wireless internet access. This development opened a new direction for wireless communication to provide Universal personal and multimedia communication irrespective of the mobility or location. These services need to be provided with high data rates. To obtain this objective future wireless networks required to support a wide range of services including voice, data, facsimile, image and video.

Because of multipath fading, Inter Symbol Interference (ISI) is introduced in the received signal for mobile radio system through Radio channel. A strong equalizer is required to eliminate the ISI from signal. The design of equalizer requires knowledge of frequency selectivity, Channel Impulse Response (CIR) [1] to mitigate the ISI.
In multiuser, a multi carrier communication system, MIMO communication channels rapidly varies due to the mobility of user. Channel State Information (CSI) is available at transmitter or receiver with feedback in the communication system. This CSI introduces problems in the radio communication system. To overcome with this problems Channel equalization techniques are Introduced [2].

Equalizers need to learn the channel characteristics (training) with Impulse response. The knowledge of impulse response is useful to estimate the frequency response if channel changes continuously (tracking). The Adaptive equalization technique includes training and tracking.

In training process, the training sequence has been sent to the adaptive equalizer at the receiver. The training sequence is a binary signal which is either pseudorandom or fixed bit pattern. The adaptive equalizer at the receiver uses mathematical algorithm to estimate filter coefficients. This estimated filter coefficients, are modified to eliminate the noise which is created due to multipath fading effect of the channel. The training sequence along with user data is sent to the receiver. If the channel is transmitting with delay spread, deepest fades the training sequence is designed to allow the equalizer at the receiver to get exact filter coefficients. Once training sequence transmission finished, filter coefficients are optimized to receive user data [8].

Equalizer tracks the time varying characteristics of the channel continuously with recursive algorithm after receiving the user data. As a result adaptive equalizer rapidly changes its filter coefficients over time. The convergence of equalizer is obtained with proper training. Convergence time of equalizer is depends on equalizer recursive algorithm, structure of the equalizer and rate of change of multipath fading channel [4].

Equalizer design must balance the removal of ISI with noise enhancement because noise and signal passes through the equalizer, so that noise power is also increases. This noise enhancement is very less in the non Linear equalizers but design of non linear equalizer 
is complex process. Linear equalizers are simple to implement. The Linear and Non Linear equalizers are implemented with lattice or traversal structure. The traversal structure of $\mathrm{N}$-coefficient filter consists with $\mathrm{N}-1$ taps, N-1 delay elements with complex weights. The structure of lattice filter is complex [3]. Adaptive equalizer requires recursive algorithm to update filter coefficients along with filter structure. In this paper equalization techniques for MIMO channels with linear equalizer are discussed. There are five adaptive linear equalizers. These are

1. Zero Forcing (ZF) equalization

2. Minimum Mean Square Error (MMSE) equalization

3. Zero Forcing equalization with Successive Interference Cancellation (ZF-SIC)

4. ZFSIC with optimal ordering.

\section{MIMO SYSTEM MODEL}

A MIMO Communication System is developed with $n T$ ransmit and $n R$ receive antennas shown in fig2.1 In this paper 2x2 MIMO systems are discussed with $n T=n R .=2$

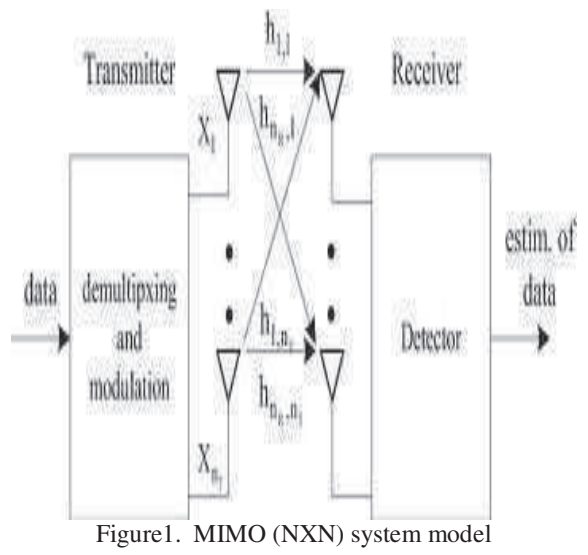

A flat fading MIMO channel is assumed with $n T$ transmit and $n R$ receive antennas. These antennas are represented by a channel matrix $(\mathrm{H})$ with $\mathrm{nR} \times n T$ antennas. The elements of $\mathbf{H}$ are independent to each other and $H j, i \in C N(0,1)$. The column vector $\mathrm{X}$ of is an input to the channel and channel output, AWGN Channel is represented by $n R \times 1$ column vectors with $\mathbf{n}$ and y respectively. The elements of $\mathbf{n}$ are assumed to be independent and $n j \in C N(0, \sigma 2)$. For convenience, we define

$\mathrm{m}=\min (n T, n R), n_{-}=\max (n T, n R), d_{-}=n-m$.

The channel input/output equation for channel can be written as

$\mathrm{Y}=\mathrm{Hx}+\mathrm{n}$
Where $\mathrm{Y}$ is the receive vector and $\mathrm{x}$ is the transmit vectors and $H$ is the channel matrix and $n$ is the noise vector respectively.

To represent $2 \times 2$ MIMO transmission channel a 2 x 2 matrix is taken with four complex-valued elements. Based on known pilot or training sequence the channel matrix elements are estimated. The signal at the receiver can be represented with

$\left(\begin{array}{c}R x O \\ R x 1 \\ \cdot \\ R \times N\end{array}\right)=\left(\begin{array}{cccc}h_{1,1} & h_{2,1} & \cdot & h_{N, 1} \\ h_{1,2} & h_{2,2} & \cdot & h_{N, 2} \\ \cdot & \cdot & \cdot & \cdot \\ h_{1, N} & h_{2, N} & \cdot & h_{N, N}\end{array}\right)\left(\begin{array}{c}T x O \\ T x 1 \\ \cdot \\ T x N\end{array}\right)$

Where

$H(t)=\left(\begin{array}{ccc}h_{1,1}(t) & \ldots & h_{1, m}(t) \\ \ldots & \ldots & \ldots \\ h_{n, 1}(t) & \ldots & h_{n, m}(t)\end{array}\right)$

Rx0 To RxN, are the received symbols

$\mathrm{h}_{1,1}$ is the fading coefficient from $1^{\text {st }}$ transmit antenna to1st receive antenna,

$\mathrm{h}_{1,2}$ is the fading coefficient from $1^{\text {st }}$ transmit antenna to $2^{\text {nd }}$ receive antenna,

$\mathrm{h}_{2,1}$ is the fading coefficient from $2^{\text {nd }}$ transmit antenna to $1^{\text {st }}$ receive antenna,

$\mathrm{h}_{2,2}$ is the fading coefficient from $2^{\text {nd }}$ transmit antenna to $2^{\text {nd }}$ receive antenna,

Tx0 to TxN are the transmitted symbols and noise is represented

$y(k, n)=\sum_{u=1}^{U} H_{u}^{(c)}(k, n) S_{u}(k, n)+\eta(k, n)$

$\eta(k, n)$ noise on receive vectors.[4]

\section{ADAPTIVE EQUALIZER}

If the time varying properties of communication channel is adapted automatically then equalizer is called adaptive equalizer.

Adaptive equalizers are implemented in the receiver either at the base band or at IF since channel response, base band signal, and demodulated signal represented with base band envelope expression. This adaptive equalizers are simulated and implemented at the base band receiver.

The original transmitter information signal represented with $\mathrm{x}(\mathrm{t})$ and combined complex base band channel impulse of the transmitter is represented with $\mathrm{f}(\mathrm{t})$. Base band noise signal at the channel input is represented with $\mathrm{nb}(\mathrm{t})$, and signal received by equalizer can be represented by equation.

$Y(t)=x(t) \otimes f(t)+n_{b}(t)$

Where $\mathrm{f}^{*}(\mathrm{t})$ denotes complex conjugate of $\mathrm{f}(\mathrm{t})$, and $*$ denotes the convolution operation. If the impulse response of the equalizer is heq $(t)$, then the output of equalizer is

$d(t)=x(t) \otimes f(t) \otimes h_{e q}(t)+n_{b}(t) \otimes h_{e q(t)}$ 
$=h(t) \otimes g(t)+n_{b}(t) \otimes h_{e q}(t)$

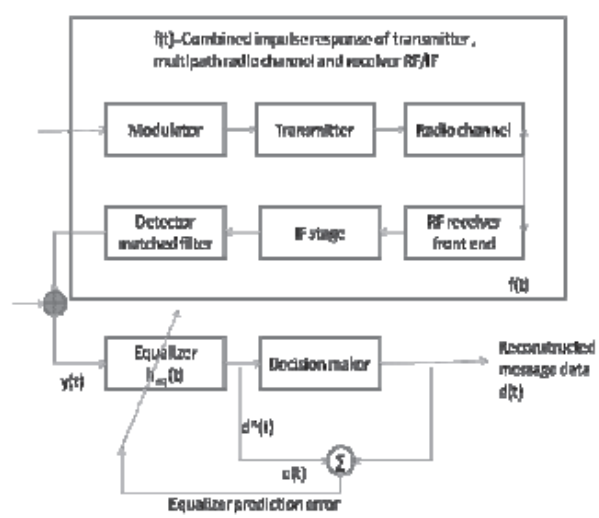

Figure2. Block diagram of a communication system with an adaptive equalizer in the receiver.

Where $\mathrm{g}(\mathrm{t})$ is the combined response of the transmitter, channel ,RF/IF sections of the receiver, and the equalizer at the receiver. The complex base band impulse response of the traversal filter equalizer is given by

$h_{e q}(t)=\sum_{n} c_{n} \delta(t-n T)$

Where $\mathrm{cn}$ is the complex filter coefficient of the equalizer. The desired output of the equalizer is $x(t)$, the original source data. Assume that $n b(t)$. Then, in order to force $\mathrm{d}(\mathrm{t})=\mathrm{x}(\mathrm{t}), \mathrm{g}(\mathrm{t})$ must be equal to

$g(t)=f(t) \otimes h_{e q}(t)=\delta(t)$

The Objective of equalization is to satisfy the given equation

$$
H_{e q}(f) F(-f)=1
$$

Where Heq (f) and F (f) are Fourier transforms of heq $(\mathrm{t})$ and $\mathrm{f}(\mathrm{t})$ respectively, so that the combination of the transmitter, channel, and receiver appear to be an all pass channel [5].

\section{A. Zero forcing $(Z F)$ Equalizer}

Zero Forcing Equalizer uses a linear equalizer algorithm for communication system. The linear equalizers are implemented based on inverse frequency response of the channel.

The name Zero Forcing corresponds to bringing down the ISI to zero in a noise free case. This will be useful when ISI is significant compared to noise. The zero frequency equalizer frequency response $C(f)$ and Channel frequency response $F(f)$ is constructed such that $C(f)=1 / F(f)$. Thus the combination of channel and equalizer gives a flat frequency response and linear phase $F(f) C(f)=1$.If the channel response for a particular channel is $\mathrm{H}(\mathrm{s})$ then the input signal is multiplied by the reciprocal of this. This is intended to remove the effect of channel from the received signal, in particular the Inter symbol Interference (ISI).

For $2 \times 2$ MIMO Channel the received signal at the first receive antenna is represented as

$y_{1}-h_{,}, x_{1}+h_{1,2} x_{2}+n_{1}-\left[h_{1,1} h_{1,2}\right]\left[\begin{array}{l}x_{1} \\ x_{2}\end{array}\right]+n_{1}$

The received signal on the second receive antenna is,

$$
y_{2}=h_{2,1} x_{1}+h_{2,2} x_{2}+n_{2}=\left[h_{2,1} h_{2,2}\right]\left[\begin{array}{l}
x_{1} \\
x_{2}
\end{array}\right]+\mathrm{n}_{2}
$$

The equation can be represented in matrix notation as follows:

$$
\left(\begin{array}{l}
y_{1} \\
y_{2}
\end{array}\right)=\left(\begin{array}{ll}
h_{1,1} & h_{1,2} \\
h_{2,1} & h_{2,2}
\end{array}\right)\left(\begin{array}{l}
x_{1} \\
x_{2}
\end{array}\right)+\left(\begin{array}{l}
n_{1} \\
n_{2}
\end{array}\right)
$$

Equivalently,

$\mathrm{y}=\mathrm{H} x+\mathrm{n}$

To solve for $x$, we need to find a matrix which satisfies $W H=I$. The Zero Forcing $(\mathrm{ZF})$ detector for meeting this constraint is given by,

$\mathrm{W}=\left(\mathrm{H}^{\mathrm{H}} \mathrm{H}\right)^{-1} \mathrm{H}^{\mathrm{H}}$

Where W - Equalization Matrix and

$\mathrm{H}$ - Channel Matrix

This matrix is known as the Pseudo inverse for a general $\mathrm{m} \times \mathrm{n}$ matrix where

$H^{H} H=\left(\begin{array}{ll}h_{1,1}^{*} & h_{1,2}^{*} \\ h_{2,1}^{*} & h_{2,2}^{*}\end{array}\right)\left(\begin{array}{ll}h_{1,1} & h_{1,2} \\ h_{2,1} & h_{2,2}\end{array}\right)=\left(\begin{array}{ll}\left|h_{1,1}\right|^{2}+\left|h_{2,1}\right|^{2} & h_{1,1}^{*} h_{1,2}+h_{2,1}^{*} h_{2,2} \\ h_{1,2}^{*} h_{1,1}+h_{2,2}^{*} h_{2,1} & \left|h_{1,2}\right|^{2}+\left|h_{2,2}\right|^{2}\end{array}\right)$

The off diagonal elements in the matrix $\mathrm{H}^{\mathrm{H}} \mathrm{H}$ are not zero, because the off diagonal elements are non zero in values. Zero forcing equalizer tries to null out the interfering terms when performing the equalization, i.e. when solving for $x 2$ the interference from $x 1$ is tried to be nulled and vice versa. During this process noise is also amplified. Hence the Zero forcing equalizer is not the best possible equalizer. However, it is simple and reasonably easy to implement. For BPSK Modulation in Rayleigh fading channel, the BER is defined as

$$
\mathrm{P}_{\mathrm{b}}=\frac{1}{2}\left(1-\sqrt{\frac{\left(E_{b} / N_{a}\right.}{\left(E_{b} / N_{a}\right)+1}}\right)
$$

Where

$\mathrm{Pb}$ - Bit Error Rate

$\mathrm{Eb} / \mathrm{No}$ - Signal to noise Ratio

\section{B. Minimum Mean Square Error (MMSE) Equalizer}

A minimum mean square error (MMSE) estimator minimizes the mean square error (MSE), which is a common measure of estimator quality. MMSE equalizer does not eliminate ISI completely but, minimizes the total power of the noise and ISI components in the output. Let $x$ be an unknown random variable, and let $y$ be a known random variable. An estimator $x^{\wedge}(\mathrm{y})$ is any function of the measurement $y$, and its mean square error is given by

$\mathrm{MSE}=\mathrm{E}\left\{\left(\mathrm{X}^{\wedge}-\mathrm{X} 2\right)\right\}$ 
where the expectation is taken over both $x$ and $y$. The Minimum Mean Square Error (MMSE) approach tries to find a coefficient $W$ which minimizes the

$$
\text { E }\left\{\left[W_{y-x}\right]\left[W_{y-x}\right]^{H}\right\}
$$

where W - Equalization Matrix

$\mathrm{H}$ - Channel Matrix and

$\mathrm{n}$ - Channel noise $\mathrm{y}$ - Received signal. To solve for $x$, we need to find a matrix $W$ which satisfies $W H=I$. The Minimum Mean Square Error (MMSE) detector for meeting this constraint is given by,

$$
\mathrm{W}=\left[\mathrm{H}^{\mathrm{H}} \mathrm{H}+\mathrm{N}_{\mathrm{o}} \mathrm{I}\right)^{-1} \mathrm{H}^{\mathrm{H}}
$$

This matrix is known as the pseudo inverse for a general $\mathrm{m} \times \mathrm{n}$ matrix.

When comparing the equation (9) with the equation (11) in Zero Forcing equalizer, apart from $N_{o} I$ the term both the equations are comparable. If the noise term is zero, the MMSE equalizer reduces to Zero Forcing equalizer.

\section{Zero Forcing with Successive Interference Cancellation (ZF-SIC)}

From the Zero Forcing (ZF) equalization approach, the receiver can obtain an estimate of the two transmitted symbols $\mathrm{x}_{1}, \mathrm{x}_{2}$

$$
\left[\begin{array}{l}
\hat{x}_{1} \\
\hat{x}_{2}
\end{array}\right]=\left(\boldsymbol{F I}^{\boldsymbol{H}} \boldsymbol{H}\right)^{-1} \boldsymbol{H} \boldsymbol{F}\left[\begin{array}{l}
y_{1} \\
y_{2}
\end{array}\right]
$$

Take one of the estimated symbols (for example $\mathrm{x} 2$ ) and subtract its effect from the received vector $\mathrm{y}_{1}$ and $\mathrm{y}$ 2

$$
\begin{aligned}
& {\left[\begin{array}{l}
r_{1} \\
r_{2}
\end{array}\right]=\left[\begin{array}{ll}
y_{1}-h_{1,2} & \widehat{x}_{2} \\
y_{2}-h_{2,2} & \widehat{x}_{2}
\end{array}\right]=\left[\begin{array}{ll}
h_{1,1} & x_{1}+n_{1} \\
h_{2,1} & x_{1}+n_{2}
\end{array}\right]} \\
& r=x_{1}+r
\end{aligned}
$$

The above equation is same as equation obtained for receive diversity case. In Maximum Ratio Combining (MRC) the received symbols are combined by taking the information from multiple copies.

$$
\hat{x}_{1}=\frac{h^{H}{ }_{r}}{h^{H} h}
$$

This forms Zero Forcing Equalizer with Successive Interference Cancellation (ZF-SIC) approach.

\section{Successive Interference Cancellation with Optimal Ordering}

In successive Interference cancellation either $\mathrm{x}_{1}$ or $\mathrm{x}_{2}$ selected first and subtracted from $\mathrm{y}_{1}$ or $\mathrm{y}_{2}$. But to make that decision, let us find out the transmit symbol (after multiplication with the channel) which came at higher power at the receiver. The received power at the both the antennas corresponding to the transmitted symbol is $\mathrm{x}_{1}$ is,

$$
P x_{1}=\left|h_{1,1}\right|^{2}+\left|h_{2,1}\right|^{2}
$$

The received power at the both the antennas corresponding to the transmitted symbol $\mathrm{x} 2$ is
$P x_{2}=\left|h_{1,2}\right|^{2}+\left|h_{2,2}\right|^{2}$

If $\mathrm{Px}_{1}>\mathrm{Px}_{2}$ then the receiver decides to remove the effect of $x_{1}$ from the received vector $y_{1}$ and $y_{2}$ then reestimate $\mathrm{x}_{2 .}$

$\left[\begin{array}{l}r_{1} \\ r_{2}\end{array}\right]=\left[\begin{array}{ll}y_{1}-h_{1,1} & x_{1} \\ y_{2}-h_{1,2} & x_{1}\end{array}\right]=\left[\begin{array}{ll}h_{1,2} & x_{2}+n_{1} \\ h_{2,2} & x_{2}+n_{2}\end{array}\right]$

expressing in matrix notation,

$$
\left[\begin{array}{l}
r_{1} \\
r_{2}
\end{array}\right]=\left[\begin{array}{l}
h_{1,2} \\
h_{2,2}
\end{array}\right] x_{2}+\left[\begin{array}{l}
n_{1} \\
n_{2}
\end{array}\right]
$$

$r=h x_{2}+n$

with Maximal Ratio Combining [6] (MRC) The equalized symbol for ${ }_{x_{2}}^{\wedge}$ is,

$\hat{x}_{2}=\frac{h^{H}{ }_{r}}{h^{H} h}$

else if $\mathrm{Px}_{1}<\mathrm{Px}_{2}$ the receiver decides to subtract effect of $\mathrm{x}_{2}$ from the received vector $\mathrm{y}_{1}$ and $\mathrm{y}_{2}$, and then reestimate expressing in $\mathrm{x}_{1}$.

$\left[\begin{array}{l}r_{1} \\ r_{2}\end{array}\right]=\left[\begin{array}{ll}y_{1}-h_{1,2} & \widehat{x}_{2} \\ y_{2}-h_{2,2} & \widehat{x}_{2}\end{array}\right]=\left[\begin{array}{ll}h_{1,1} x_{1}+n_{1} \\ h_{2,1} x_{1}+n_{2}\end{array}\right]$

Expressing in matrix notation,

$$
\left[\begin{array}{l}
r_{1} \\
r_{2}
\end{array}\right]=\left[\begin{array}{l}
h_{1,1} \\
h_{2,1}
\end{array}\right] x_{1}+\left[\begin{array}{l}
n_{1} \\
n_{2}
\end{array}\right]
$$

$r=h x_{1}+n$

And the equalized symbol $\mathrm{x} 1$ with Maximal Ratio Combining [6] (MRC). is,

$$
x_{1}=\frac{h_{r}^{H}}{h^{H} h}
$$

Doing successive interference cancellation with optimal ordering ensures that the reliability of the symbol which is decoded first is guaranteed to have a lower error probability than the other symbol. This results in lowering the chances of incorrect decisions resulting in erroneous interference cancellation. Hence gives lower error rate than simple successive interference cancellation. [7]

\section{RESUlTS}

\section{A. Zero forcing $(Z F)$ Equalizer}

The zero-forcing equalizer removes all ISI, and is ideal when the channel is noiseless. However, when the channel is noisy, the zero-forcing equalizer will amplify the noise greatly at frequencies $f$ where the channel response $\mathrm{H}(\mathrm{j} 2 \pi f)$ has a small magnitude (i.e. near zeroes of the channel) in the attempt to invert the channel completely.

From simulation results, Zero forcing equalizers improve the data rate, but not diversity gain. 


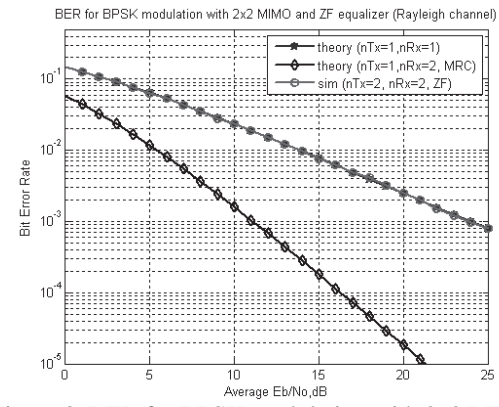

Figure 3. BER for BPSK modulation with $2 \times 2$ MIMO and $\mathrm{ZF}$ equalizer

\section{B. Minimum Mean Square Error (MMSE) Equalizer}

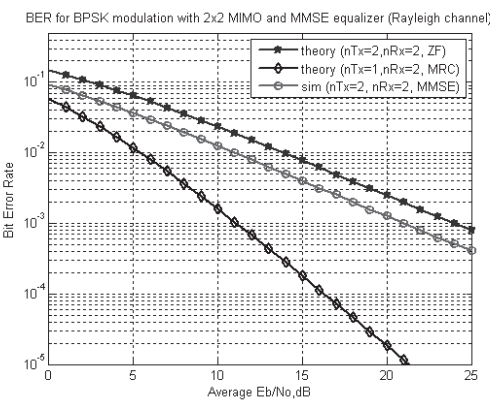

Figure 4. BER for BPSK modulation with $2 \times 2$ MIMO and MMSE equalizer

Minimum Mean Square Error (MMSE) equalizer results in around $3 \mathrm{~dB}$ of improvement of BER at $10^{-3}$

\section{Zero Forcing with Successive Interference Cancellation (ZF-SIC)}

Compare to Zero forcing equalizer, with successive Interference Cancellation 3.2dB improvement in BER.

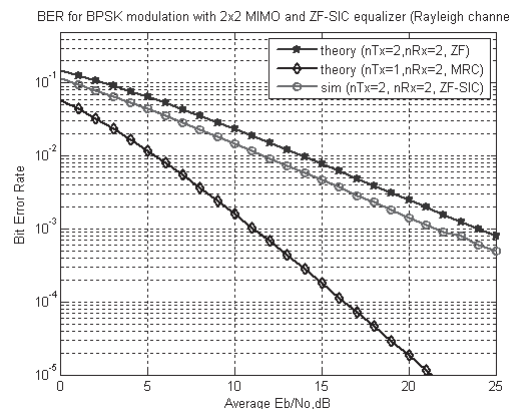

Figure4. BER for BPSK modulation with 2x2 MIMO and ZF SIC equalizer

\section{Successive Interference Cancellation with optimal ordering (SIC).}

Compared to Minimum Mean Square Equalization with simple successive interference cancellation case, addition of optimal ordering results in around $5.0 \mathrm{~dB}$ BER improvement

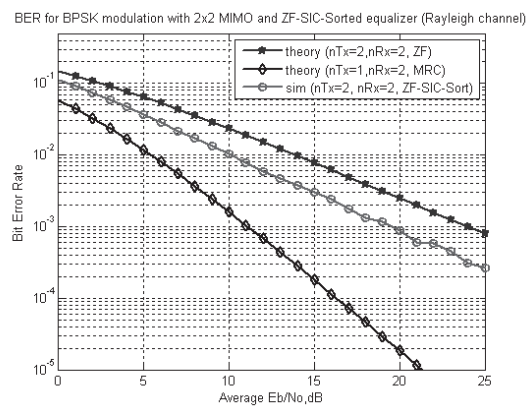

Figure 5. BER for BPSK modulation with $2 \times 2$ MIMO and ZF SIC Optimized equalizer

\section{CONCLUSIONS}

Due to mobility, multiuser, multicarrier and time varying property of Channels, MIMO requires equalization techniques for wireless communication. In this paper Equalization technique are compared for MIMO. From simulation results we can conclude that MMSE with SIC can cancel out interference with optimum level.

\section{REFERENCES}

[1] Ardavn malekhi- Tehrani,Babak Hasibi, John M. coeffi 'frequency selective channels Adaptive equalization techniques of Multi Input Multi Output' IEEE Transaction. Wireless Communication 1999.

[2] Sajid Ahmeda, Sangarapillai Lambotharan, Andreas Jakobsson, Jonathon Chambers of' Parameter estimation 'and equalization techniques for MIMOfrequency selective channels with multiple frequency offsets.

[3] Zero-forcing equalization for timevaryingsystem with memory"byCassio B. Ribeiro, L. R. de Campos, and Paulo S.R.Diniz.

[4] zero-forcing frequency domain equalization for DMT systems within sufficient guard interval by Tanja Karp , Martin J. Wolf , Steffen Trautmann, and Norbert J. Fliege.

[5] Jun Shi Huiyu Luo Yu-Ching Tong, Adaptive Equalizer for MIMO WirelessCommunication Channels March 12, 2003.

[6] O. Simeone, Y. Bar-Ness, and U.Spagnolini, Linear and Nonlinear Preequalization/Equalization for MIMO SystemsWith Long-Term Channel State Information at the Transmitter, IEEE transactions on wireless communications, vol. 3, no. 2, march 2004.

[7] 'Wireless Communications' by F.Molish

[8] Wireless Communication, by "GoldSmith" 